\title{
Stuart S. Antman \\ Addendum: \\ Existence and Nonuniqueness \\ of Axisymmetric Equilibrium States \\ of Nonlinearly Elastic Shells
}

Vol. 40, pp. 329-372 (1971)

The author is grateful to Dr. R. J. ElliotT and Dr. J. D. M. WRIGHT for their reading of the manuscript.

Courant Institute

New York University

(Received March 18, 1971) 